Pacific

Journal of

Mathematics

\title{
TAUT FOLIATIONS IN SURFACE BUNDLES WITH MULTIPLE BOUNDARY COMPONENTS
}

TEJAs KALELKAR AND RACHEL RobeRTS

Volume 273 No. 2

February 2015 


\title{
TAUT FOLIATIONS IN SURFACE BUNDLES WITH MULTIPLE BOUNDARY COMPONENTS
}

\author{
TEJas KALELKar AND RACHEl Roberts
}

\begin{abstract}
Let $M$ be a fibered 3-manifold with multiple boundary components. We show that the fiber structure of $M$ transforms to closely related transversely oriented taut foliations realizing all rational multislopes in some open neighborhood of the multislope of the fiber. Each such foliation extends to a taut foliation in the closed 3-manifold obtained by Dehn filling along its boundary multislope. The existence of these foliations implies that certain contact structures are weakly symplectically fillable.
\end{abstract}

\section{Introduction}

Any closed, orientable 3-manifold can be realized by Dehn filling a 3-manifold which is fibered over $S^{1}$ [Alexander 1920; Myers 1978]. In other words, any closed oriented 3-manifold can be realized by Dehn filling a 3-manifold $M_{0}$, where $M_{0}$ has the form of a mapping torus

$$
M_{0}=S \times[0,1] / \sim,
$$

where $S$ is a compact orientable surface with nonempty boundary and $\sim$ is an equivalence relation given by $(x, 1) \sim(h(x), 0)$ for some orientation-preserving homeomorphism $h: S \rightarrow S$ which fixes the components of $\partial S$ setwise. Although we shall not appeal to this fact in this paper, it is interesting to note that it is possible to assume that $h$ is pseudo-Anosov [Colin and Honda 2008] and hence $M_{0}$ is hyperbolic [Thurston 1988]. It is also possible to assume that $S$ has positive genus. Any nonorientable closed 3-manifold admits a double cover of this form.

Taut codimension-one foliations are topological objects which have proved very useful in the study of 3-manifolds. The problem of determining when a 3-manifold contains a taut foliation appears to be a very difficult one. A complete classification exists for Seifert fibered manifolds [Brittenham 1993; Eisenbud et al. 1981; Jankins and Neumann 1985; Naimi 1994], but relatively little is known for the case of hyperbolic 3-manifolds. There are many partial results demonstrating existence

MSC2010: 57M50.

Keywords: Dehn filling, taut foliation, fibered 3-manifold, contact structure, open book decomposition. 
(see, for example, [Calegari and Dunfield 2003; Delman and Roberts 1999; Gabai 1983; 1987a; 1987b; Li 2002; Li and Roberts 2013; Roberts 1995; 2001a; 2001b]) and partial results demonstrating nonexistence [Jun 2004; Kronheimer and Mrowka 2007; Kronheimer et al. 2007; Roberts et al. 2003]. In this paper, we investigate the existence of taut codimension-one foliations in closed orientable 3-manifolds by first constructing taut codimension-one foliations in corresponding mapping tori $M_{0}$. In contrast with [Roberts 2001a; 2001b], we consider the case that the boundary of $M_{0}$ is not connected. We obtain the following results. Precise definitions will follow in Section 2.

Theorem 1.1. Given an orientable, fibered compact 3-manifold, a fibration with fiber surface of positive genus can be modified to yield transversely oriented taut foliations realizing a neighborhood of rational boundary multislopes about the boundary multislope of the fibration.

As an immediate corollary for closed manifolds we therefore have:

Corollary 1.2. Let $M=\widehat{M}_{0}\left(r^{j}\right)$ be the closed manifold obtained from $M_{0}$ by Dehn filling $M_{0}$ along the multicurve with rational multislope $\left(r^{j}\right)_{j=1}^{k}$. When $\left(r^{j}\right)$ is sufficiently close to the multislope of the fibration, $M$ admits a transversely oriented taut foliation.

Dehn filling $M_{0}$ along the slope of the fiber gives a mapping torus of a closed surface with the fibration as the obvious taut foliation. This corollary shows that Dehn filling $M_{0}$ along slopes sufficiently close to the multislope of the fiber also gives a closed manifold with a taut foliation.

When the surgery coefficients $r^{j}$ are all meridians, the description of $M$ as a Dehn filling of $M_{0}$ gives an open book decomposition $(S, h)$ of $M$. The foliations of Corollary 1.2 can be approximated by a pair of contact structures, one positive and one negative, both naturally related to the contact structure $\xi_{(S, h)}$ compatible with the open book decomposition $(S, h)$ [Eliashberg and Thurston 1998; Kazez and Roberts 2014]. It follows that the contact structure $\xi_{(S, h)}$ is weakly symplectically fillable.

Corollary 1.3. Let $M$ have open book decomposition $(S, h)$. Then $M$ is obtained by Dehn filling $M_{0}$ along the multicurve with rational multislope $\left(r^{j}\right)_{j=1}^{k}$, where the $r^{j}$ are all meridians. When $\left(r^{j}\right)$ is sufficiently close to the multislope of the fibration, $\xi_{(S, h)}$ is weakly symplectically fillable and hence universally tight.

It is natural to ask whether the qualifier "sufficiently close" can be made precise.

Honda, Kazez, and Matić [Honda et al. 2008] proved that when an open book with connected binding has monodromy with fractional Dehn twist coefficient $c$ at least one, it supports a contact structure which is close to a coorientable taut foliation. Note that $c \geq 1$ is sufficient but not always necessary to guarantee that $\xi_{(S, h)}$ is close to a coorientable taut foliation. 
For an open book with multiple binding components, there is no such global lower bound on the fractional Dehn twist coefficients sufficient to guarantee that $\xi_{(S, h)}$ is close to a coorientable taut foliation. This was shown by Baldwin and Etnyre [2013], who constructed a sequence of open books with arbitrarily large fractional Dehn twist coefficients and disconnected bindings that support contact structures which are not deformations of a taut foliation. So we cannot expect to obtain a neighborhood around the slope of the fiber which would satisfy our criteria of "sufficiently close" for every open book decomposition. At the end of the paper, in Section 4, we explicitly compute a neighborhood around the multislope of the fiber realizable by our construction for the Baldwin-Etnyre examples.

\section{Preliminaries}

In this section we introduce basic definitions and fix conventions used in the rest of the paper.

Foliations. Roughly speaking, a codimension-one foliation $\mathscr{F}$ of a 3-manifold $M$ is a disjoint union of injectively immersed surfaces such that $(M, \mathscr{F})$ looks locally like $\left(\mathbb{R}^{3}, \mathbb{R}^{2} \times \mathbb{R}\right)$.

Definition 2.1. Let $M$ be a closed $C^{\infty}$ 3-manifold and let $r$ be a nonnegative integer or infinity. A $C^{r}$ codimension-one foliation $\mathscr{F}$ of (or in) $M$ is a union of disjoint connected surfaces $L_{i}$, called the leaves of $\mathscr{F}$, in $M$ such that

(1) $\bigcup_{i} L_{i}=M$, and

(2) there exists a $C^{r}$ atlas $\mathscr{A}$ on $M$ which contains all $C^{\infty}$ charts and with respect to which $\mathscr{F}_{p}$ satisfies the following local product structure: for every $p \in M$, there exists a coordinate chart $(U,(x, y, z))$ in $\mathscr{A}$ about $p$ such that $U \approx \mathbb{R}^{3}$ and the restriction of $\mathscr{F}_{F}$ to $U$ is the union of planes given by $z=$ constant.

When $r=0$, we require also that the tangent plane field $T \mathscr{F}$ be $C^{0}$.

A taut foliation [Gabai 1983] is a codimension-one foliation of a 3-manifold for which there exists a transverse simple closed curve that has nonempty intersection with each leaf of the foliation. Although every 3-manifold contains a codimensionone foliation [Lickorish 1965; Novikov 1965; Wood 1969], it is not true that every 3-manifold contains a codimension-one taut foliation. In fact, the existence of a taut foliation in a closed orientable 3-manifold has important topological consequences for the manifold. For example, if $M$ is a closed, orientable 3-manifold that has a taut foliation with no sphere leaves then $M$ is covered by $\mathbb{R}^{3}$ [Palmeira 1978], $M$ is irreducible [Rosenberg 1968] and has an infinite fundamental group [Haefliger 1962]. In fact, its fundamental group acts nontrivially on interesting 1-dimensional objects (see, for example, [Thurston 1998; Calegari and Dunfield 2003; Palmeira 


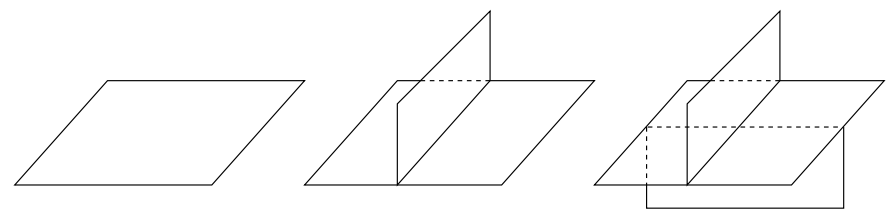

Figure 1. Local model of a standard spine.

1978; Roberts et al. 2003]). Moreover, taut foliations can be perturbed to weakly symplectically fillable contact structures [Eliashberg and Thurston 1998] and hence can be used to obtain Heegaard-Floer information [Ozsváth and Szabó 2004].

Multislopes. Let $F$ be a compact oriented surface of positive genus and with nonempty boundary consisting of $k$ components. Let $h$ be an orientation-preserving homeomorphism of $F$ which fixes each boundary component pointwise. Let

$$
M=F \times I /(x, 1) \sim(h(x), 0),
$$

and denote the $k$ (toral) boundary components of $\partial M$ by $T^{1}, T^{2}, \ldots, T^{k}$.

We use the given surface bundle structure on $M$ to fix a coordinate system on each of the boundary tori, as follows. (See, for example, [Rolfsen 1976, Section 9.G] for a definition and description of this coordinate system.) For each $j$ we choose as longitude $\lambda^{j}=\partial F \cap T^{j}$, with orientation inherited from the orientation of $F$. For each $j$, we then fix as meridian $\mu_{j}$ an oriented simple closed curve dual to $\lambda_{j}$. Although, as described in [Kazez and Roberts 2014; Roberts 2001b], it is possible to use the homeomorphism $h$ to uniquely specify such simple closed curves $\mu_{j}$, we choose not to do so in this paper, as all theorem statements are independent of the choice of meridional multislope.

We say a taut foliation $\mathscr{F}_{F}$ in $M$ realizes boundary multislope $\left(m^{j}\right)_{j=1}^{k}$ if for each $j, 1 \leq j \leq k, \mathscr{F} \cap T^{j}$ is a foliation of $T^{j}$ of slope $m^{j}$ in the chosen coordinate system of $T^{j}$.

\section{Spines and branched surfaces.}

Definition 2.2. A standard spine [Casler 1965] is a space $X$ locally modeled on one of the spaces of Figure 1. The critical locus of $X$ is the 1-complex of points of $X$ where the spine is not locally a manifold.

Definition 2.3. A branched surface (see [Williams 1974] and [Oertel 1984; 1988]) is a space $B$ locally modeled on the spaces of Figure 2. The branching locus $L$ of $B$ is the 1-complex of points of $B$ where $B$ is not locally a manifold. The components of $B \backslash L$ are called the sectors of $B$. The points where $L$ is not locally a manifold are called double points of $L$. 


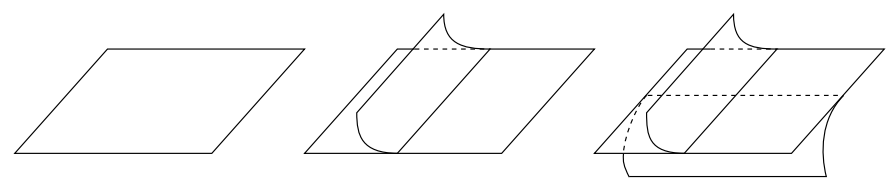

Figure 2. Local model of a branched surface.

A standard spine $X$ together with an orientation in a neighborhood of the critical locus determines a branched surface $B$ in the sense illustrated in Figure 3.

Example 2.4. Let $F_{0}:=F \times\{0\}$ be a fiber of $M=F \times I /(x, 1) \sim(h(x), 0)$. Let $\alpha_{i}, 1 \leq i \leq k$, be pairwise disjoint, properly embedded arcs in $F_{0}$, and set $D_{i}=\alpha_{i} \times I$ in $M$. Isotope the image $\operatorname{arcs} h\left(\alpha_{i}\right)$ as necessary so that the intersection $\left(\bigcup \alpha_{i}\right) \cap\left(\bigcup_{i} h\left(\alpha_{i}\right)\right)$ is transverse and minimal. Assign an orientation to $F$ and to each $D_{i}$. Then $X=F_{0} \cup \bigcup_{i} D_{i}$ is a transversely oriented spine. We will denote by $B=\left\langle F ; \bigcup_{i} D_{i}\right\rangle$ the transversely oriented branched surface associated with $X$.

Similarly, $\left\langle\bigcup_{i} F_{i} ; \bigcup_{i, j} D_{i}^{j}\right\rangle$ will denote the transversely oriented branched surface associated to the transversely oriented standard spine

$$
X=F_{0} \cup F_{1} \cup \cdots \cup F_{n-1} \cup \bigcup_{i, j} D_{i}^{j},
$$

where $F_{i}=F \times\{i / n\}$ and $D_{i}^{j}=\alpha_{i}^{j} \times[i / n,(i+1) / n]$ for some set of $\operatorname{arcs} \alpha_{i}^{j}$ properly embedded in $F$ so that the intersection $\left(\bigcup_{j} \alpha_{i-1}^{j}\right) \cap\left(\bigcup_{j} \alpha_{i}^{j}\right)$ is transverse and minimal.

Definition 2.5. A lamination carried by a branched surface $B$ in $M$ is a closed subset $\lambda$ of an $I$-fibered regular neighborhood $N(B)$ of $B$, such that $\lambda$ is a disjoint union of injectively immersed 2-manifolds (called leaves) that intersect the $I$-fibers of $N(B)$ transversely.

Laminar branched surfaces. Li [2002; 2003] introduced the fundamental notions of sink disk and half sink disk.

Definition 2.6 [Li 2002; 2003]. Let $B$ be a branched surface in a 3-manifold $M$. Let $L$ be the branching locus of $B$ and let $X$ denote the union of double points of $L$. Associate to each component of $L \backslash X$ a vector (in $B$ ) pointing in the direction of the cusp. A sink disk is a disk branch sector $D$ of $B$ for which the branch direction
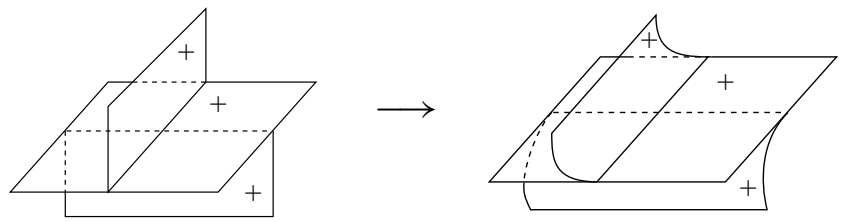

Figure 3. Oriented spine to oriented branched surface. 


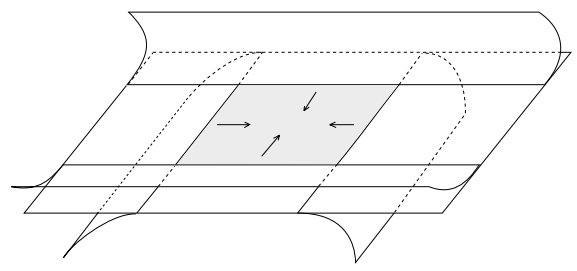

Figure 4. A sink disk.

of each component of $(L \backslash X) \cap \bar{D}$ points into $D$ (as shown in Figure 4). A half sink disk is a sink disk which has nonempty intersection with $\partial M$.

Sink disks and half sink disks play a key role in Li's notion of laminar branched surface.

Definition 2.7 [Li 2002, Definition 1.3]. Let $D_{1}$ and $D_{2}$ be the two disk components of the horizontal boundary of a $D^{2} \times I$ region in $M \backslash$ int $N(B)$. If the projection $\pi: N(B) \rightarrow B$ restricted to the interior of $D_{1} \cup D_{2}$ is injective, that is, the intersection of any $I$-fiber of $N(B)$ with int $D_{1} \cup$ int $D_{2}$ is either empty or a single point, then we say that $\pi\left(D_{1} \cup D_{2}\right)$ forms a trivial bubble in $B$.

Definition 2.8 [Li 2002, Definition 1.4]. A branched surface $B$ in a closed 3-manifold $M$ is called a laminar branched surface if it satisfies the following conditions:

(1) $\partial_{h} N(B)$ is incompressible in $M \backslash$ int $N(B)$, no component of $\partial_{h} N(B)$ is a sphere and $M \backslash B$ is irreducible.

(2) There is no monogon in $M \backslash$ int $N(B)$, that is, no disk $D \subset M \backslash$ int $N(B)$ with $\partial D=D \cap N(B)=\alpha \cup \beta$, where $\alpha \subset \partial_{v} N(B)$ is in an interval fiber of $\partial_{v} N(B)$ and $\beta \subset \partial_{h} N(B)$

(3) There is no Reeb component; that is, $B$ does not carry a torus that bounds a solid torus in $M$.

(4) $B$ has no trivial bubbles.

(5) $B$ has no sink disk or half sink disk.

Gabai and Oertel [1989] introduced essential branched surfaces and proved that any lamination fully carried by an essential branched surface is an essential lamination and, conversely, any essential lamination is fully carried by an essential branched surface. In practice, to check if a manifold has an essential lamination, the tricky part often is to verify that a candidate branched surface does in fact fully carry a lamination. Li [2002] uses laminar branched surfaces to relax this requirement and prove the following:

Theorem 2.9 [Li 2002, Theorem 1]. Suppose that M is a closed and orientable 3-manifold. Then: 
(1) Every laminar branched surface in M fully carries an essential lamination.

(2) Any essential lamination in $M$ that is not a lamination by planes is fully carried by a laminar branched surface.

Li [2003] noticed that if a branched surface has no half sink disk, then it can be arbitrarily split in a neighborhood of its boundary train track without introducing any sink disk (or half sink disk). He was therefore able to conclude the following.

Theorem 2.10 [Li 2003, Theorem 2.2]. Let $M$ be an irreducible and orientable 3-manifold whose boundary is a union of incompressible tori. Suppose B is a laminar branched surface and $\partial M \backslash \partial B$ is a union of bigons. Then, for any multislope $\left(s_{1}, \ldots, s_{k}\right) \in(\mathbb{Q} \cup\{\infty\})^{k}$ that can be realized by the train track $\partial B$, if $B$ does not carry a torus that bounds a solid torus in $\widehat{M}\left(s_{1}, \ldots, s_{k}\right)$, then $B$ fully carries a lamination $\lambda_{\left(s_{1}, \ldots, s_{k}\right)}$ whose boundary consists of the multislope $\left(s_{1}, \ldots, s_{k}\right)$, and $\lambda_{\left(s_{1}, \ldots, s_{k}\right)}$ can be extended to an essential lamination in $\widehat{M}\left(s_{1}, \ldots, s_{k}\right)$.

We note that Li stated Theorem 2.2 only for the case that $\partial M$ is connected. However, as Li has observed and is easily seen, his proof extends immediately to the case that $\partial M$ consists of multiple toral boundary components. Key is the fact that splitting $B$ open, to a branched surface $B^{\prime}$ say, in a neighborhood of its boundary, so that $\partial B^{\prime}$ consists of multislopes $\left(s_{1}, \ldots, s_{k}\right)$, does not introduce sink disks. Therefore, capping $B^{\prime}$ off to $\widehat{B}^{\prime}$ yields a laminar branched surface in $\widehat{M}\left(s_{1}, \ldots, s_{k}\right)$.

Good oriented sequence of arcs. In this section we introduce some definitions that will be used in the rest of the paper.

Definition 2.11. Let $\left(\alpha^{1}, \ldots, \alpha^{k}\right)$ be a tuple of pairwise disjoint simple arcs properly embedded in $F$ with $\partial \alpha^{j} \subset T^{j}$. Such a tuple will be called parallel if $F \backslash\left\{\alpha^{1}, \ldots, \alpha^{k}\right\}$ has $k$ components, $k-1$ of which are annuli $\left\{A^{j}\right\}$ with $\partial A^{j}$ containing $\left\{\alpha^{j}, \alpha^{j+1}\right\}$ and one of which is a surface $S$ of genus $g-1$ with $\partial S$ containing $\left\{\alpha^{1}, \alpha^{k}\right\}$. Furthermore, all $\alpha^{j}$ are oriented in parallel, that is, the orientation of $\partial A^{j}$ agrees with $\left\{-\alpha^{j}, \alpha^{j+1}\right\}$ and the orientation of $\partial S$ agrees with $\left\{-\alpha^{k}, \alpha^{1}\right\}$. Note that, in particular, each $\alpha^{j}$ is nonseparating. See Figure 5 for an example of a parallel tuple.

Definition 2.12. A pair of tuples $\left(\alpha^{i}\right)_{i=1, \ldots, k}$ and $\left(\beta^{j}\right)_{j=1, \ldots, k}$ will be called good if both are parallel tuples and $\alpha^{i}$ and $\beta^{j}$ have exactly one (interior) point of intersection when $i \neq j$, while $\alpha^{i}$ is disjoint from $\beta^{j}$ when $i=j$.

A sequence of parallel tuples

$$
\sigma=\left(\left(\alpha_{0}^{1}, \alpha_{0}^{2}, \ldots, \alpha_{0}^{k}\right),\left(\alpha_{1}^{1}, \alpha_{1}^{2}, \ldots, \alpha_{1}^{k}\right), \ldots,\left(\alpha_{n}^{1}, \alpha_{n}^{2}, \ldots, \alpha_{n}^{k}\right)\right),
$$

also shortened to

$$
\left(\left(\alpha_{0}^{j}\right),\left(\alpha_{1}^{j}\right), \ldots,\left(\alpha_{n}^{j}\right)\right)
$$




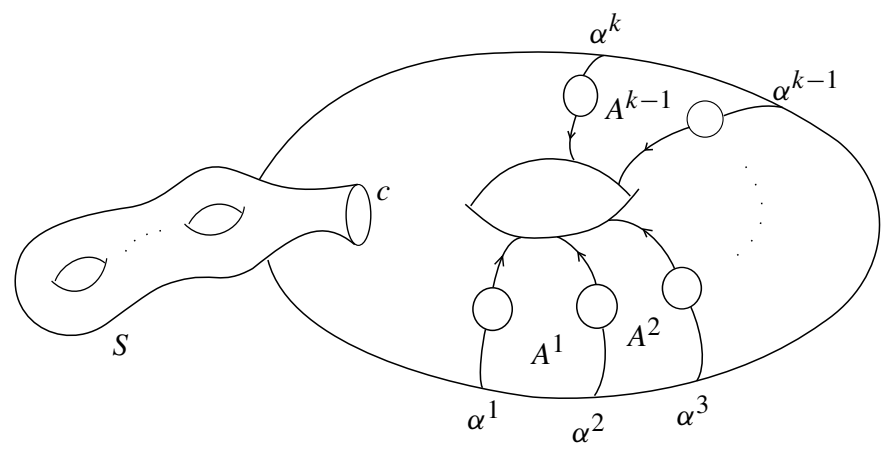

Figure 5. A parallel tuple $\left(\alpha^{i}\right)$ on the surface $F$.

or

$$
\left(\alpha_{0}^{j}\right) \stackrel{\sigma}{\rightarrow}\left(\alpha_{n}^{j}\right)
$$

will be called good if, for each fixed $j, 1 \leq j \leq k$, the pair $\left(\left(\alpha_{i}^{j}\right),\left(\alpha_{i+1}^{j}\right)\right)$ is good.

Definition 2.13. We say a good pair $\left(\left(\alpha^{j}\right),\left(\beta^{j}\right)\right)$ is positively oriented if for each $j \in\{1, \ldots, k\}$ a neighborhood of the $j$-th boundary component in $F$ is as shown on the right in Figure 6. Similarly, we say a good pair $\left(\left(\alpha^{j}\right),\left(\beta^{j}\right)\right)$ is negatively oriented if for each $j \in\{1, \ldots, k\}$ a neighborhood of the $j$-th boundary component in $F$ is as shown on the left in Figure 6.

We say a good sequence $\sigma=\left(\left(\alpha_{0}^{j}\right),\left(\alpha_{1}^{j}\right), \ldots,\left(\alpha_{n}^{j}\right)\right)$ is positively oriented if each pair $\left(\left(\alpha_{i}^{j}\right),\left(\alpha_{i+1}^{j}\right)\right)$ is positively oriented. Similarly, $\sigma=\left(\left(\alpha_{1}^{j}\right),\left(\alpha_{2}^{j}\right), \ldots,\left(\alpha_{n}^{j}\right)\right)$ is negatively oriented if each pair $\left(\left(\alpha_{i}^{j}\right),\left(\alpha_{i+1}^{j}\right)\right)$ is negatively oriented. We say the sequence $\sigma$ is oriented if it is positively or negatively oriented. See Figure 7 for an example of a negatively oriented good pair in $F$.

Preferred generators. Let

$$
\begin{aligned}
\mathscr{H}_{g, k}=\left\{\eta_{1}, \eta_{2}, \ldots, \eta_{2 g-2+k,},\right. & \gamma_{12}, \gamma_{24}, \gamma_{46}, \gamma_{68}, \ldots, \\
& \left.\gamma_{2 g-4,2 g-2}, \beta, \beta_{1}, \beta_{2}, \ldots, \beta_{g-1}, \delta_{1}, \delta_{2}, \ldots, \delta_{k-1}\right\}
\end{aligned}
$$
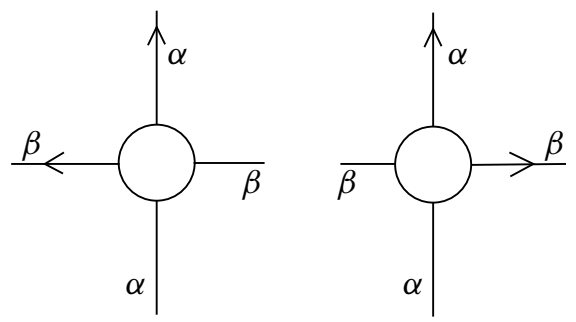

Figure 6. Left: a negatively oriented pair of $\operatorname{arcs}(\alpha, \beta)$. Right: a positively oriented pair of $\operatorname{arcs}(\alpha, \beta)$. 


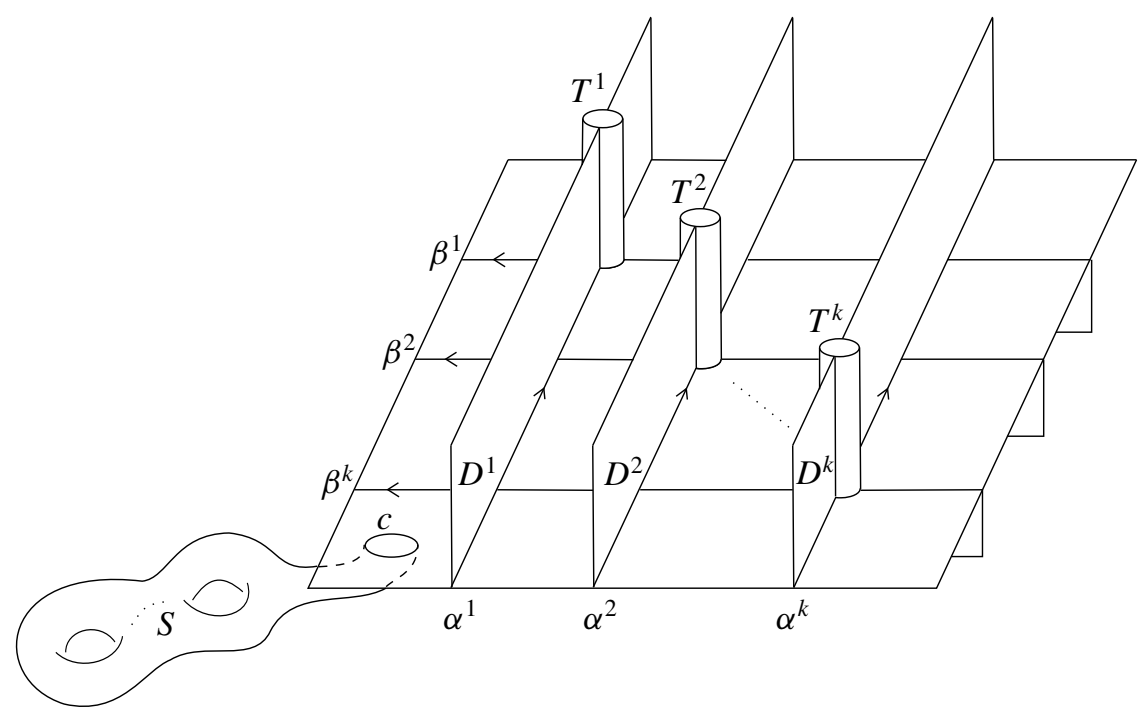

Figure 7. Neighborhood of $F$ with a good negatively oriented pair $\left(\left(\alpha^{j}\right),\left(\beta^{j}\right)\right)$ in the oriented spine $X$.

be the curves on $F$ as shown in Figure 8. Then by [Gervais 2001, Proposition 1 and Theorem 1] the mapping class group $\operatorname{MCG}(F, \partial F)$ of $F$ (fixing boundary) is generated by Dehn twists about curves in $\mathscr{H}_{g, k}$.

Theorem (Gervais). The mapping class group $\operatorname{MCG}(F, \partial F)$ of $F$ is generated by Dehn twists about the curves in $\mathscr{H}_{g, k}$.

As Dehn twists about $\delta_{i}$ are isotopic to the identity via an isotopy that does not fix the boundary, we have the following obvious corollary:

Corollary 2.14. The mapping class group $\mathrm{MCG}(F)$ of $F$ (not fixing the boundary pointwise) is generated by Dehn twists about the curves in

$$
\mathscr{H}_{g, k}^{\prime}=\mathscr{H}_{g, k} \backslash\left\{\delta_{1}, \ldots, \delta_{k-1}\right\} .
$$

\section{Main theorem}

Definition 3.1. Let $\left(\alpha^{1}, \ldots, \alpha^{k}\right)$ be a parallel tuple in $F$. Orient $F$ so that the normal vector $\hat{n}$ induced by the orientation of $M$ points in the direction of increasing $t \in[0,1]$. Let $D^{j}=\alpha^{j} \times[0,1]$ in $M_{h}$ with the orientation induced by orientations of $\alpha^{j}$ and $F$; that is, if $v^{j}$ is tangent to $\alpha^{j}$ then $\left(v^{j}, \hat{n}\right)$ gives the orientation of $D^{j}$. Let $X=F \cup \bigcup_{j} D^{j}$ be an oriented standard spine and $B_{\alpha}=\left\langle F ; \bigcup_{j} D^{j}\right\rangle$ the transversely oriented branched surface associated with $X$.

Notice that the multislope of the fibration is $\overline{0}$. In order to prove Theorem 1.1, we shall prove the following: 


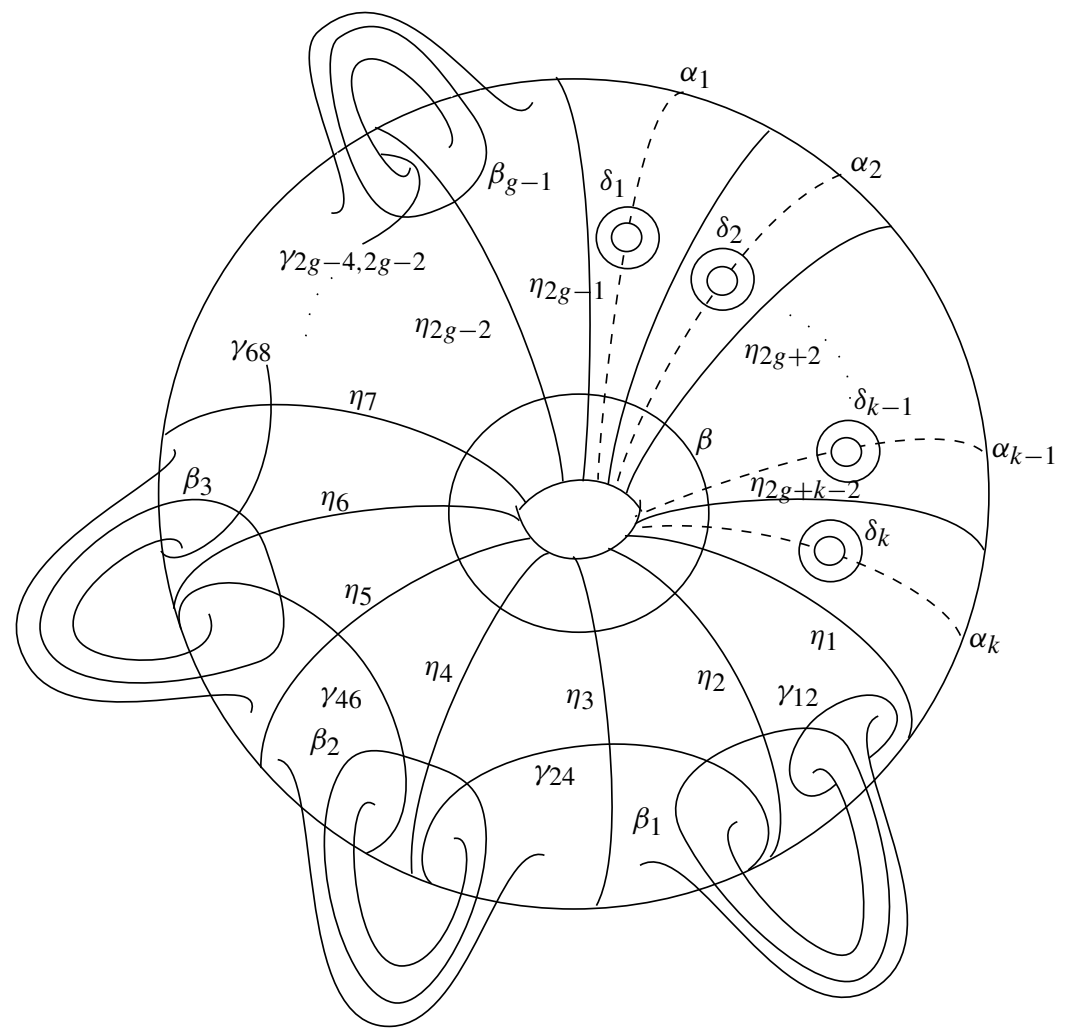

Figure 8. Generators of the mapping class group.

Theorem 3.2. There is an open neighborhood $U$ of $\overline{0} \in \mathbb{R}^{k}$ such that, for each point $\left(m^{1}, \ldots, m^{k}\right) \in U \cap \mathbb{Q}^{k}$, there exists a lamination carried by $B_{\alpha}$ with boundary multislope $\left(\mathrm{m}^{j}\right)$. These laminations extend to taut foliations which also intersect the boundary in foliations with multislope $\left(\mathrm{m}^{j}\right)$.

This gives us the following corollary for closed manifolds.

Corollary 3.3. Let $\widehat{M}\left(r^{j}\right)$ denote the closed manifold obtained from $M$ by a Dehn filling along a multicurve with rational multislope $\left(r^{j}\right)_{j=1}^{k}$. For each tuple $\left(r^{j}\right)$ in $U \cap \mathbb{Q}^{k}$, the closed manifold $\widehat{M}\left(r^{j}\right)$ also has a transversely oriented taut foliation.

We outline the proof of Theorem 3.2 with details worked out in the lemmas.

Proof. In Lemma 3.4 we show that there is a good positively oriented sequence $\left(\alpha_{0}^{j}\right) \rightarrow\left(h^{-1}\left(\alpha_{0}^{j}\right)\right)$, or equivalently from $\left(h\left(\alpha_{n}^{j}\right)\right) \rightarrow\left(\alpha_{n}^{j}\right)$. In Lemma 3.6 we show that whenever there exists such a positive sequence there is a splitting of the branched surface $B_{\alpha}$ to a branched surface $B_{\sigma}$ that is laminar and that therefore carries laminations realizing every multislope in some open neighborhood of $\overline{0} \in \mathbb{R}^{k}$. Finally, in Lemma 3.8 we show that these laminations extend to taut foliations on all of $M$. 
Lemma 3.4. Let $\left(\alpha^{j}\right)$ be a parallel tuple in $F$ and let $h \in \operatorname{Aut}^{+}(F)$. Then there is a good positively oriented sequence $\left(\alpha^{j}\right) \stackrel{\sigma}{\rightarrow}\left(h\left(\alpha^{j}\right)\right)$.

Proof. By Corollary 2.14 to Gervais's theorem, $h \sim h_{m} h_{m-1} \cdots h_{2} h_{1}$ for twists $h_{i}$ about curves in $\mathscr{H}_{g, k}^{\prime}$. Set $h^{\prime}=h_{m} h_{m-1} \cdots h_{2} h_{1}$, and notice that $M_{h}=M_{h^{\prime}}$.

By changing the handle decomposition of $F$ as necessary, we may assume that the parallel tuple $\left(\alpha^{j}\right)$ is as shown in Figure 8. Let $b$ denote the Dehn twist about $\beta \in \mathscr{H}_{g, k}^{\prime}$. Notice that any $h_{i}$ in the factorization of $h^{\prime}$ is either $b, b^{-1}$ or a twist about a curve disjoint from all components of $\alpha^{j}$. Thus $\left(\left(\alpha^{j}\right),\left(h_{i}\left(\alpha^{j}\right)\right)\right.$ is either a good positive pair, a good negative pair, or a pair of equal tuples.

Now, if $\left(\left(\alpha^{j}\right),\left(\beta^{j}\right)\right)$ is a good pair then so is $\left(\left(h_{i}\left(\alpha^{j}\right)\right),\left(h_{i}\left(\beta^{j}\right)\right)\right)$; therefore, each of the pairs

$$
\begin{aligned}
& \left(\left(\alpha^{j}\right), h_{m}\left(\alpha^{j}\right)\right), \\
& \left(\left(h_{m}\left(\alpha^{j}\right)\right),\left(h_{m} h_{m-1}\left(\alpha^{j}\right)\right)\right), \\
& \left(\left(h_{m} h_{m-1}\left(\alpha^{j}\right)\right),\left(h_{m} h_{m-1} h_{m-2}\left(\alpha^{j}\right)\right)\right), \\
& \quad \vdots \\
& \left(\left(h_{m} h_{m-1} \cdots h_{2}\left(\alpha^{j}\right)\right),\left(h_{m} h_{m-1} \cdots h_{2} h_{1}\left(\alpha^{j}\right)=h\left(\alpha^{j}\right)\right)\right),
\end{aligned}
$$

is either a good oriented pair or a pair of equal tuples.

If at least one of the $h_{i}$ is $b$ or $b^{-1}$ then, ignoring the equal tuples, we get a good oriented sequence $\left(\left(\alpha_{0}^{j}\right),\left(\alpha_{1}^{j}\right), \ldots,\left(\alpha_{n-1}^{j}\right),\left(\alpha_{n}^{j}\right)=h\left(\left(\alpha_{0}^{j}\right)\right)\right)$ or $\left(\alpha^{j}\right) \stackrel{\sigma}{\rightarrow}\left(h\left(\alpha^{j}\right)\right)$ as required. The length of this sequence is equal to the number of times $h_{i}$ equals $b$ or $b^{-1}$, that is, $n=n_{+}+n_{-}$, where $n_{+}$is the sum of the positive powers of $b$ in this expression of $h^{\prime}$ and $n_{-}$is the magnitude of the sum of negative powers of $b$.

If none of the $h_{i}$ are Dehn twists about $\beta$ then $\left(\alpha^{j}\right)=\left(h\left(\alpha^{j}\right)\right)$. In this case, $\sigma=\left(\left(\alpha^{j}\right),\left(b\left(\alpha^{j}\right)\right),\left(b^{-1} b\left(\alpha^{j}\right)=\left(\alpha^{j}\right)\right)\right)$ is a good oriented sequence.

If $\left(\left(\alpha^{j}\right),\left(\beta^{j}\right)\right)$ is a positively oriented good pair then $\left(\left(\alpha^{j}\right),\left(-\beta^{j}\right),\left(-\alpha^{j}\right),\left(\beta^{j}\right)\right)$ is a negatively oriented good sequence. Performing $n_{-}$such substitutions, we get a positively oriented good sequence $\left(\alpha^{j}\right) \stackrel{\sigma}{\rightarrow}\left(h\left(\alpha^{j}\right)\right)$.

Definition 3.5. Let $\sigma=\left(h\left(\alpha_{n}^{j}\right)=\alpha_{0}^{j}, \alpha_{1}^{j}, \ldots, \alpha_{n-1}^{j}, \alpha_{n}^{j}\right)$ be a good oriented sequence. Let $F_{i}=F \times\{i / n\}$ for $0 \leq i<n$ and let $D_{i}^{j}=\alpha_{i}^{j} \times[i / n,(i+1) / n]$, for $0 \leq i<n$, in $M_{h}$. Let

$$
X=\left(\bigcup_{i} F_{i}\right) \cup\left(\bigcup_{i, j} D_{i}^{j}\right),
$$

and orient $F_{i}$ and $D_{i}^{j}$ as in Definition 3.1. Define

$$
B_{\sigma}=\left\langle\bigcup_{i} F_{i} ; \bigcup_{i, j} D_{i}^{j}\right\rangle
$$

as the associated branched surface. Figure 7 shows the neighborhood of $F$ in $X$, while Figure 9 shows a neighborhood of $F$ in the associated branched surface. 


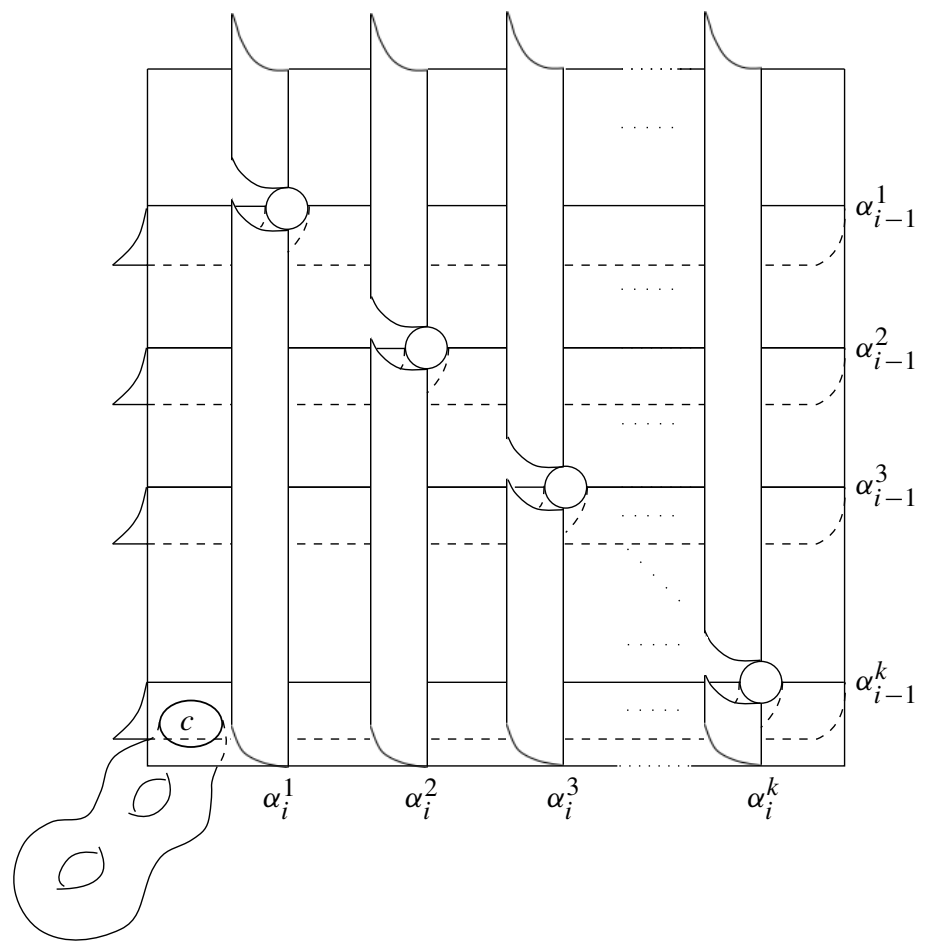

Figure 9. A neighborhood of one of the fibers in the branched surface $B$. The small circles along the diagonal represent longitudes of the boundary tori. The vertical subarcs of the boundaries of the vertical disk sectors lie on these boundary tori. Compare with Figure 7.

Lemma 3.6. Let $\sigma=\left(h\left(\alpha_{n}^{j}\right)=\alpha_{0}^{j}, \alpha_{1}^{j}, \ldots, \alpha_{n-1}^{j}, \alpha_{n}^{j}\right)$ be a good oriented sequence in $F$ and $B_{\sigma}$ the associated branched surface in $M_{h}$. Then $B_{\sigma}$ has no sink disk or half sink disk.

Proof. As the sequence $\sigma$ is good and oriented for each fixed $i$, the tuple of arcs $\left(\alpha_{i}^{j}\right)$ is parallel and $\left|\alpha_{i}^{j} \cap \alpha_{i-1}^{k}\right|=\delta_{j}^{k}$, so a neighborhood of $F_{i}$ in $B_{\sigma}$ is as shown in Figure 9.

The sectors of $B_{\sigma}$ consist of disks $D_{i}^{j}=\alpha_{i}^{j} \times[i / n,(i+1) / n]$ and components of $F_{i} \backslash\left\{\alpha_{i}^{j} \cup \alpha_{i-1}^{j}\right\}_{j=1, \ldots, k}$. As $F_{i-1}$ and $F_{i}$ both have a coorientation in the direction of increasing $t$ for $(x, t) \in M_{h}$, so for any orientation of $D_{i}^{j}, \partial D_{i}^{j}$ is the union of two arcs in $\partial M_{h}$, together with one arc with the direction of the cusp pointing into the disk and one arc with the direction of the cusp pointing outwards. Similarly, as $\alpha_{i}^{j}$ and $\alpha_{i}^{j+1}$ are oriented in parallel, each disk component of $F_{i} \backslash\left\{\alpha_{i}^{j}, \alpha_{i-1}^{j}\right\}_{j=1, \ldots, k}$ has a boundary arc with cusp direction pointing outwards. Therefore, no branch sector in $B_{\sigma}$ is a sink disk or a half sink disk. 


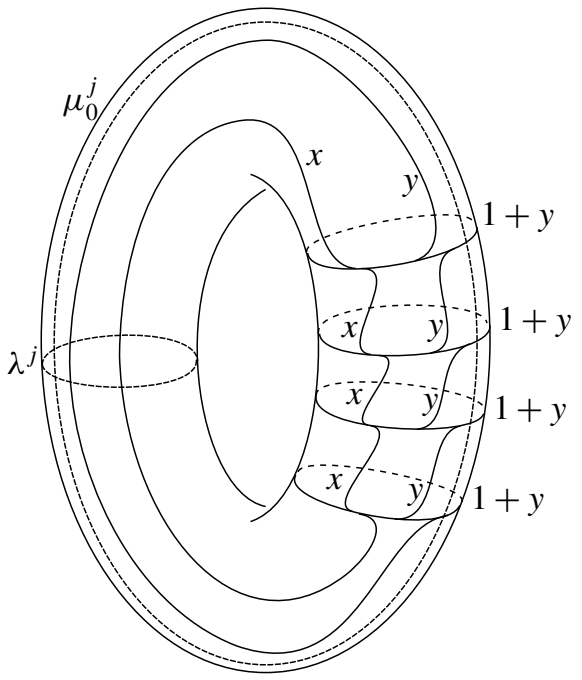

Figure 10. The weighted boundary train track when $n=4$.

Remark 3.7. Notice that $B_{\sigma}=\left\langle\bigcup_{i} F_{i} ; \bigcup_{i, j} D_{i}^{j}\right\rangle$ is a splitting (see [Oertel 1988]) of the original branched surface $B_{\alpha}=\left\langle F ; \bigcup_{j} D^{j}\right\rangle$ and, equivalently, $B_{\sigma}$ collapses to $B_{\alpha}$. So, in particular, laminations carried by $B_{\sigma}$ are also carried by $B_{\alpha}$.

Now consider the train tracks $\tau^{j}=B_{\sigma} \cap T^{j}$. Focus on one of the $\tau^{j}$. Recall that we fixed a coordinate system $\left(\lambda^{j}, \mu^{j}\right)$ on $T^{j}$. For simplicity of exposition, we now make a second choice $\mu_{0}^{j}$ of meridian. This choice is dictated by the form of $\tau^{j}$; namely, we choose $\mu_{0}^{j}$ to be disjoint from the disks $D_{i}^{j}$ so that $\tau^{j}$ has the form shown in Figure 10. Notice that there is a change of coordinates homeomorphism taking slopes in terms of the coordinate system $\left(\lambda^{j}, \mu_{0}^{j}\right)$ to slopes in terms of the coordinate system $\left(\lambda^{j}, \mu^{j}\right)$. Since $\lambda^{j}$ is unchanged, this homeomorphism takes an open interval about 0 to an open interval about 0 . Assign to $\tau^{j}$ the measure determined by weights $x, y$ shown in Figure 10. In terms of the coordinate system $\left(\lambda^{j}, \mu_{0}^{j}\right), \tau^{j}$ carries all slopes realizable by

$$
\frac{x-y}{n(1+y)}
$$

for some $x, y>0$. Therefore, in terms of the coordinate system $\left(\lambda^{j}, \mu_{0}^{j}\right), \tau^{j}$ carries all slopes in $(-1 / n, \infty)$. Converting to the coordinate system $\left(\lambda^{j}, \mu^{j}\right), \tau^{j}$ carries all slopes in some open neighborhood of 0 . Repeat for all $j$. By Theorem 2.10, we see that the branched surface $B_{\sigma}$ carries laminations $\lambda_{(\bar{x}, \bar{y})}$ realizing multislopes

$$
\left(\frac{x_{1}-y_{1}}{n\left(1+y_{1}\right)}, \frac{x_{2}-y_{2}}{n\left(1+y_{2}\right)}, \ldots, \frac{x_{k}-y_{k}}{n\left(1+y_{k}\right)}\right)
$$


for any strictly positive values of $x_{1}, \ldots, x_{k}, y_{1}, \ldots, y_{k}$, and hence realizing all rational multislopes in some open neighborhood of $\overline{0} \in \mathbb{R}^{k}$.

Lemma 3.8. Suppose the weights $\bar{x}, \bar{y}$ are distinct and have strictly positive coordinates. Then each lamination $\lambda_{(\bar{x}, \bar{y})}$ contains only noncompact leaves. Furthermore, each lamination $\lambda_{(\bar{x}, \bar{y})}$ extends to a taut foliation $\mathscr{F}_{(\bar{x}, \bar{y})}$, which realizes the same multislope.

Proof. Suppose that $\lambda_{(\bar{x}, \bar{y})}$ contains a compact leaf $L$. Such a leaf determines a transversely invariant measure on $B$ given by counting intersections with $L$.

Now focus on any $i, j$, where $0 \leq i, j<n$. By considering, for example, a simple closed curve in $F_{i}$ parallel to the arc $\alpha_{i}^{j}$, we see that there is an oriented simple closed curve in $F_{i}$ which intersects the branching locus of $B_{\sigma}$ exactly $k$ times and which has orientation consistent with the branched locus. Since this is true for all possible $i, j$, it follows that the only transversely invariant measure $B$ can support is the one with all weights on the branches $D_{i}^{j}$ necessarily 0 . But this means that $\lambda_{(\bar{x}, \bar{y})}$ realizes multislope $\overline{0}$ and hence that $\bar{x}=\bar{y}$.

The complementary regions to the lamination $\lambda_{(\bar{x}, \bar{y})}$ are product regions. Filling these up with product fibrations, we get the required foliation $\left.\mathscr{F}_{(\bar{x}}, \bar{y}\right)$, which also has no compact leaves and is therefore taut.

\section{Example}

As discussed in the introduction, an open book with connected binding and monodromy with fractional Dehn twist coefficient more than one supports a contact structure which is the deformation of a coorientable taut foliation [Honda et al. 2008]. However, for open books with disconnected binding there is no such universal lower bound on the fractional Dehn twist coefficient. This was illustrated by Baldwin and Etnyre [2013] who constructed a sequence of open books with arbitrarily large fractional Dehn twist coefficients and disconnected binding that support contact structures which are not deformations of a taut foliation. This shows, in particular, that there is no global neighborhood about the multislope of the fiber of a surface bundle such that Dehn filling along rational slopes in that neighborhood produces closed manifolds with taut foliations.

The notion of "sufficiently close" in Corollary 1.2 can, however, be bounded below for a given manifold. Deleting a neighborhood of the binding in the BaldwinEtnyre examples gives a surface bundle, and using the techniques developed in the previous sections we now calculate a neighborhood of multislopes realized by taut foliations around the multislope of the fiber in this fibration. In particular, we observe that this neighborhood does not contain the meridional multislope. So Dehn filling along these slopes does not give a taut foliation of the sequence of Baldwin-Etnyre manifolds, as is to be expected. 


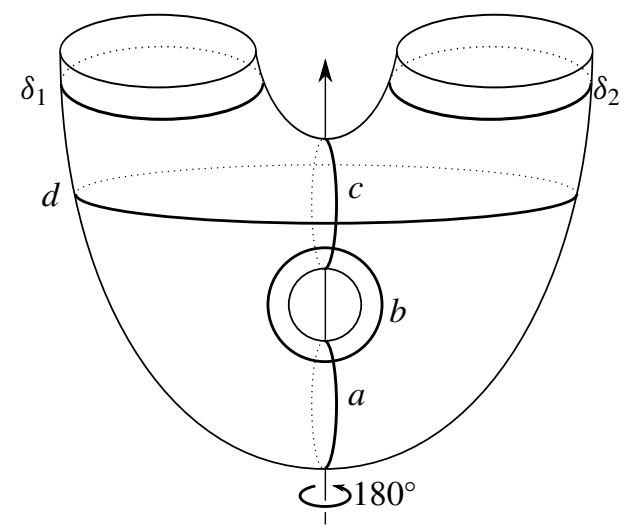

Figure 11. The Baldwin-Etnyre examples.

The following is a description of the Baldwin-Etnyre examples [2013]. Let $T$ denote the genus one surface with two boundary components, $B_{1}$ and $B_{2}$. Let $\psi$ be the diffeomorphisms of $T$ given by the product of Dehn twists,

$$
\psi=D_{a} D_{b}^{-1} D_{c} D_{d}^{-1},
$$

where $a, b, c$ and $d$ are the curves shown in Figure 11 (reproduced from Figure 1 of [Baldwin and Etnyre 2013]). Then $\psi$ is pseudo-Anosov by a well-known construction of Penner [1988]. We define

$$
\psi_{n, k_{1}, k_{2}}=D_{\delta_{1}}^{k_{1}} D_{\delta_{2}}^{k_{2}} \psi^{n},
$$

where $\delta_{1}$ and $\delta_{2}$ are curves parallel to the boundary components $B_{1}$ and $B_{2}$ of $T$.

Let $M_{n, k_{1}, k_{2}}$ be the open book $\left(T, \psi_{n, k_{1}, k_{2}}\right)$. Let $N\left(B_{1}\right), N\left(B_{2}\right)$ be regular neighborhoods of $B_{1}$ and $B_{2}$ in $M_{n, k_{1}, k_{2}}$ and let $M_{n, k_{1}, k_{2}}^{\prime}=M_{n, k_{1}, k_{2}} \backslash\left(N\left(B_{1}\right) \cup N\left(B_{2}\right)\right)$. Let $\lambda_{1}, \lambda_{2}$ be the closed curves in $T \cap \partial M_{n, k_{1}, k_{2}}^{\prime}$ represented by $B_{1}, B_{2}$, with induced orientation. The monodromy $\psi_{n, k_{1}, k_{2}}$ is freely isotopic to the pseudoAnosov map $\psi^{n}$. Let $\mu_{1}, \mu_{2}$ be the suspension flow of a point in $\lambda_{1}$ and $\lambda_{2}$, respectively, under the monodromy $\psi^{n}$. As $\psi^{n}$ is the identity on $\partial T, \mu_{i}=p_{i} \times S^{1}$ in $\partial M_{n, k_{1}, k_{2}}^{\prime}=\left(B_{1} \times S^{1}\right) \cup\left(B_{2} \times S^{1}\right)$ for $p_{i} \in \lambda_{i}$.

We use these pairs of dual curves $\left(\lambda_{1}, \mu_{1}\right)$ and $\left(\lambda_{2}, \mu_{2}\right)$ as coordinates to calculate the slope of curves on the boundary tori of $M_{n, k_{1}, k_{2}}^{\prime}$, as detailed in Section 2.

If $D_{1}$ is the meridional disk of a regular neighborhood $N\left(B_{1}\right)$ of $B_{1}$ in $M_{n, k_{1}, k_{2}}$, then $\partial D_{1}=\mu_{1}$. Similarly, for $D_{2}$ a meridional disk of a regular neighborhood of $B_{2}$ in $M_{n, k_{1}, k_{2}}, \partial D_{2}=\mu_{2}$.

In order to express the monodromy of the surface bundle in terms of the Gervais generators we use the pseudo-Anosov monodromy $\psi^{n}=\psi_{n, 0,0}$ which is freely isotopic to $\psi_{n, k_{1}, k_{2}}$, with the observation that Dehn filling $M_{n, 0,0}^{\prime}$ along slopes $-1 / k_{1}$ 


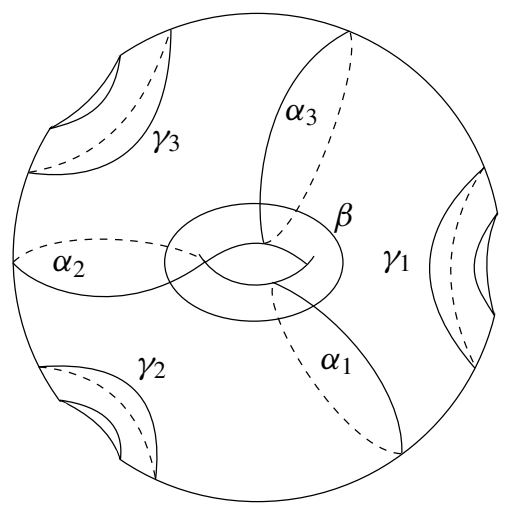

Figure 12. The Gervais star-relation.

and $-1 / k_{2}$ gives the manifold $M_{n, k_{1}, k_{1}}$. So for $M_{n, 0,0}^{\prime}$ we have slope $\left(\partial D_{1}\right)=-1 / k_{1}$, $\operatorname{slope}\left(\partial D_{2}\right)=-1 / k_{2}$.

As shown in Theorem 1.16 of [Baldwin and Etnyre 2013], for any $N>0$ there exist $n, k_{1}>N$ such that the corresponding open book in $M_{n, k_{1}, n}$ has a compatible contact structure that is not a deformation of the tangent bundle of a taut foliation. We shall now show that the slope $-1 / n$ lies outside the interval of perturbation that gives slopes of taut foliations via our construction. Hence, the manifolds $M_{n, k_{1}, n}$ cannot be obtained by capping off the taut foliations realized by our interval of boundary slopes around the fibration.

To obtain the branched surface required in our construction in the previous sections we need a good sequence of $\operatorname{arcs} \alpha^{j} \rightarrow \phi^{-1}\left(\alpha^{j}\right)$, where $\phi=\psi^{n}, j=1,2$. These arcs are used to construct product disks which we then smooth along copies of the fiber surface to get the required branched surface.

Following the method outlined in Lemma 3.4, we need to express $\phi^{-1}$ in terms of the Gervais generators. The curves $a, b$ and $c$ correspond to the generating curves $\eta_{1}, \beta$ and $\eta_{2}$ among the Gervais generators, as can be seen in Figure 8. We now need to express the curve $d$ in terms of these generating curves.

Definition 4.1. Let $S_{g, n}$ be a surface of genus $g$ and $n$ boundary components. Consider a subsurface of $S_{g, n}$ homeomorphic to $S_{1,3}$. Then for curves $\alpha_{i}, \beta, \gamma_{i}$ as shown in Figure 12 (reproduced from Figure 2 of [Gervais 2001]), the star-relation is

$$
\left(D_{\alpha_{1}} D_{\alpha_{2}} D_{\alpha_{3}} D_{\beta}\right)^{3}=D_{\gamma_{1}} D_{\gamma_{2}} D_{\gamma_{3}},
$$

where $D$ represents Dehn-twist along the corresponding curves.

Let $S$ be the component of $T \backslash d$ which is homeomorphic to a once-punctured torus. Let $\gamma_{1}=d$ and $\gamma_{2}, \gamma_{3}$ be curves bounding disjoint disks $D_{1}$ and $D_{2}$ in $S$ so 
that $S \backslash\left(D_{1} \cup D_{2}\right)$ is homeomorphic to $S_{1,3}$. As $\gamma_{2}, \gamma_{3}$ are trivial in $T, \gamma_{1}=d$ and $\alpha_{1}=\alpha_{2}=\alpha_{3}=a$, so the star relation reduces to $D_{d}=\left(D_{a}^{3} D_{b}\right)^{3}$.

Hence, the monodromy $\psi$ in terms of the Gervais generators is the word $\psi=$ $D_{a} D_{b}^{-1} D_{c}\left(D_{a}^{3} D_{b}\right)^{-3}$, which gives us $\psi^{-1}=D_{a}^{3} D_{b} D_{a}^{3} D_{b} D_{a}^{3} D_{b} D_{c}^{-1} D_{b} D_{a}^{-1}$. Take $\operatorname{arcs} \alpha_{1}, \alpha_{2}$ as shown in Figure 8, where $a=\eta_{1}, b=\beta$ and $c=\eta_{2}$. Then, as $\left(\alpha_{j}, D_{b}\left(\alpha_{j}\right)\right)$ is a negatively oriented pair and $\alpha_{j}=D_{a}\left(\alpha_{j}\right), \alpha_{j}=D_{c}\left(\alpha_{j}\right)$ so we have a negatively oriented good sequence $\left(\alpha_{1}, \alpha_{2}\right) \rightarrow\left(\psi^{-1}\left(\alpha_{1}\right), \psi^{-1}\left(\alpha_{2}\right)\right)$ obtained by taking the sequence of arcs

$$
\begin{gathered}
\sigma=\left(\alpha_{j}, D_{a}^{3} D_{b}\left(\alpha_{j}\right), D_{a}^{3} D_{b} D_{a}^{3} D_{b}\left(\alpha_{j}\right), D_{a}^{3} D_{b} D_{a}^{3} D_{b} D_{a}^{3} D_{b}\left(\alpha_{j}\right),\right. \\
\left.D_{a}^{3} D_{b} D_{a}^{3} D_{b} D_{a}^{3} D_{b} D_{c}^{-1} D_{b} D_{a}^{-1}\left(\alpha_{j}\right)=\psi^{-1}\left(\alpha_{j}\right)\right) \text { for } j=1,2 .
\end{gathered}
$$

Let $B_{\sigma}$ be the branched surface corresponding to this good oriented sequence, as in Definition 3.5. The weighted train track $\tau_{\sigma}=B_{\sigma} \cap \partial M_{n, 0,0}^{\prime}$ on the boundary tori is as shown in Figure 10.

The slope of this measured boundary lamination is $(x-y) /(4(1+y))$, so the interval of slopes that are realized by taut foliations is $\left(-\frac{1}{4}, \infty\right)$.

When the monodromy is $\psi^{n}$ (instead of $\psi$ ), by a similar argument, we get the slope of the measured lamination on the boundary as $(x-y) /(4 n(1+y))$ so that the interval of slopes realized by taut foliations is $(-1 / 4 n, \infty)$. And we observe that the point $\left(-1 / k_{1},-1 / n\right)$ does not lie in $(-1 / 4 n, \infty) \times(-1 / 4 n, \infty)$; that is, the taut foliations from our construction cannot be capped off to give a taut foliation of the Baldwin-Etnyre examples.

\section{References}

[Alexander 1920] J. W. Alexander, "Note on Riemann spaces", Bull. Amer. Math. Soc. 26:8 (1920), 370-372. MR 1560318 JFM 47.0529.02

[Baldwin and Etnyre 2013] J. A. Baldwin and J. B. Etnyre, "Admissible transverse surgery does not preserve tightness", Math. Ann. 357:2 (2013), 441-468. MR 3096514 Zbl 06228509

[Brittenham 1993] M. Brittenham, "Essential laminations in Seifert-fibered spaces", Topology 32:1 (1993), 61-85. MR 94c:57027 Zbl 0791.57013

[Calegari and Dunfield 2003] D. Calegari and N. M. Dunfield, "Laminations and groups of homeomorphisms of the circle", Invent. Math. 152:1 (2003), 149-204. MR 2005a:57013 Zbl 1025.57018

[Casler 1965] B. G. Casler, "An imbedding theorem for connected 3-manifolds with boundary", Proc. Amer. Math. Soc. 16 (1965), 559-566. MR 31 \#2730 Zbl 0129.15801

[Colin and Honda 2008] V. Colin and K. Honda, "Stabilizing the monodromy of an open book decomposition”, Geom. Dedicata 132 (2008), 95-103. MR 2008m:57041 Zbl 1151.57017

[Delman and Roberts 1999] C. Delman and R. Roberts, "Alternating knots satisfy Strong Property P", Comment. Math. Helv. 74:3 (1999), 376-397. MR 2001g:57009 Zbl 0949.57002

[Eisenbud et al. 1981] D. Eisenbud, U. Hirsch, and W. Neumann, "Transverse foliations of Seifert bundles and self-homeomorphism of the circle", Comment. Math. Helv. 56:4 (1981), 638-660. MR 83j:57016 Zbl 0516.57015 
[Eliashberg and Thurston 1998] Y. M. Eliashberg and W. P. Thurston, Confoliations, University Lecture Series 13, Amer. Math. Soc., Providence, RI, 1998. MR 98m:53042 Zbl 0893.53001

[Gabai 1983] D. Gabai, "Foliations and the topology of 3-manifolds", J. Differential Geom. 18:3 (1983), 445-503. MR 86a:57009 Zbl 0533.57013

[Gabai 1987a] D. Gabai, "Foliations and the topology of 3-manifolds, II", J. Differential Geom. 26:3 (1987), 461-478. MR 89a:57014a Zbl 0627.57012

[Gabai 1987b] D. Gabai, "Foliations and the topology of 3-manifolds, III", J. Differential Geom. 26:3 (1987), 479-536. MR 89a:57014b Zbl 0639.57008

[Gabai and Oertel 1989] D. Gabai and U. Oertel, "Essential laminations in 3-manifolds", Ann. of Math. (2) 130:1 (1989), 41-73. MR 90h:57012 Zbl 0685.57007

[Gervais 2001] S. Gervais, "A finite presentation of the mapping class group of a punctured surface", Topology 40:4 (2001), 703-725. MR 2002m:57025 Zbl 0992.57013

[Haefliger 1962] A. Haefliger, "Variétés feuilletées", Ann. Scuola Norm. Sup. Pisa (3) 16 (1962), 367-397. MR 32 \#6487 Zbl 0122.40702

[Honda et al. 2008] K. Honda, W. H. Kazez, and G. Matić, "Right-veering diffeomorphisms of compact surfaces with boundary, II”, Geom. Topol. 12:4 (2008), 2057-2094. MR 2009i:57057 Zbl 1170.57013

[Jankins and Neumann 1985] M. Jankins and W. D. Neumann, "Rotation numbers of products of circle homeomorphisms”, Math. Ann. 271:3 (1985), 381-400. MR 86g:58082 Zbl 0543.57019

[Jun 2004] J. Jun, “(-2, 3, 7)-pretzel knot and Reebless foliation”, Topology Appl. 145:1-3 (2004), 209-232. MR 2005g:57015 Zbl 1090.57014

[Kazez and Roberts 2014] W. H. Kazez and R. Roberts, "Approximating $C^{0}$-foliations", preprint, 2014. To appear in Interactions between low-dimensional topology and mapping class groups (Bonn, 2013), Geometry and Topology Monographs 19, Math. Sciences Publishers, Berkeley, 2015. arXiv $1404.5919 \mathrm{v} 2$

[Kronheimer and Mrowka 2007] P. Kronheimer and T. Mrowka, Monopoles and three-manifolds, New Mathematical Monographs 10, Cambridge University Press, 2007. MR 2009f:57049 Zbl 1158.57002

[Kronheimer et al. 2007] P. Kronheimer, T. Mrowka, P. Ozsváth, and Z. Szabó, "Monopoles and lens space surgeries", Ann. of Math. (2) 165:2 (2007), 457-546. MR 2008b:57037 Zbl 1204.57038

[Li 2002] T. Li, "Laminar branched surfaces in 3-manifolds", Geom. Topol. 6 (2002), 153-194. MR 2003h:57019 Zbl 1067.57011

[Li 2003] T. Li, "Boundary train tracks of laminar branched surfaces", pp. 269-285 in Topology and geometry of manifolds (Athens, GA, 2001), edited by G. Matić and C. McCrory, Proc. Sympos. Pure Math. 71, Amer. Math. Soc., Providence, RI, 2003. MR 2005h:57028 Zbl 1043.57006

[Li and Roberts 2013] T. Li and R. Roberts, "Taut foliations in knot complements", preprint, 2013. To appear in Pac. J. Math. arXiv 1211.3066

[Lickorish 1965] W. B. R. Lickorish, "A foliation for 3-manifolds", Ann. of Math. (2) 82 (1965), 414-420. MR 32 \#6488 Zbl 0142.41104

[Myers 1978] R. Myers, “Open book decompositions of 3-manifolds”, Proc. Amer. Math. Soc. 72:2 (1978), 397-402. MR 80a:57004 Zbl 0395.57002

[Naimi 1994] R. Naimi, "Foliations transverse to fibers of Seifert manifolds", Comment. Math. Helv. 69:1 (1994), 155-162. MR 94m:57060 Zbl 0797.55009

[Novikov 1965] S. P. Novikov, “The topology of foliations”, Trudy Moskov. Mat. Obšč. 14 (1965), 248-278. In Russian; translated in Trans. Moscow Math. Soc. 14 (1965), 268-304. MR 34 \#824 Zbl 0247.57006

[Oertel 1984] U. Oertel, "Incompressible branched surfaces", Invent. Math. 76:3 (1984), 385-410. MR 86a:57007 Zbl 0539.57006 
[Oertel 1988] U. Oertel, "Measured laminations in 3-manifolds", Trans. Amer. Math. Soc. 305:2 (1988), 531-573. MR 89d:57011 Zbl 0652.57006

[Ozsváth and Szabó 2004] P. Ozsváth and Z. Szabó, "Holomorphic disks and genus bounds", Geom. Topol. 8 (2004), 311-334. MR 2004m:57024 Zbl 1056.57020

[Palmeira 1978] C. F. B. Palmeira, “Open manifolds foliated by planes”, Ann. Math. (2) 107:1 (1978), 109-131. MR 58 \#18490 Zbl 0382.57010

[Penner 1988] R. C. Penner, "A construction of pseudo-Anosov homeomorphisms", Trans. Amer. Math. Soc. 310:1 (1988), 179-197. MR 89k:57026 Zbl 0706.57008

[Roberts 1995] R. Roberts, "Constructing taut foliations", Comment. Math. Helv. 70:4 (1995), 516545. MR 96j:57032 Zbl 0855.57009

[Roberts 2001a] R. Roberts, "Taut foliations in punctured surface bundles, I", Proc. London Math. Soc. (3) 82:3 (2001), 747-768. MR 2003a:57040 Zbl 1034.57017

[Roberts 2001b] R. Roberts, "Taut foliations in punctured surface bundles, II", Proc. London Math. Soc. (3) 83:2 (2001), 443-471. MR 2003j:57016 Zbl 1034.57018

[Roberts et al. 2003] R. Roberts, J. Shareshian, and M. Stein, "Infinitely many hyperbolic 3-manifolds which contain no Reebless foliation”, J. Amer. Math. Soc. 16:3 (2003), 639-679. MR 2004e:57023 Zbl 1012.57022

[Rolfsen 1976] D. Rolfsen, Knots and links, Mathematics Lecture Series 7, Publish or Perish, Berkeley, CA, 1976. MR 58 \#24236 Zbl 0339.55004

[Rosenberg 1968] H. Rosenberg, "Foliations by planes", Topology 7 (1968), 131-138. MR 37 \#3595 Zbl 0157.30504

[Thurston 1988] W. P. Thurston, "On the geometry and dynamics of diffeomorphisms of surfaces", Bull. Amer. Math. Soc. (N.S.) 19:2 (1988), 417-431. MR 89k:57023 Zbl 0674.57008

[Thurston 1998] W. P. Thurston, “Three-manifolds, foliations and circles, II”, Unfinished manuscript, 1998.

[Williams 1974] R. F. Williams, "Expanding attractors”, Inst. Hautes Études Sci. Publ. Math. 43 (1974), 169-203. MR 50 \#1289 Zbl 0279.58013

[Wood 1969] J. W. Wood, "Foliations on 3-manifolds", Ann. of Math. (2) 89 (1969), 336-358. MR 40 \#2123 Zbl 0176.21402

Received September 15, 2013. Revised September 23, 2013.

TEJAS KALELKAR

DEPARTMENT OF MATHEMATICS

WASHINGTON UNIVERSITY IN ST. LOUIS

1 BROOKINGS DRIVE

ST. LOUIS, MO 63130

UNITED STATES

tejas@math.wustl.edu

RACHEL ROBERTS

DEPARTMENT OF MATHEMATICS

WASHINGTON UNIVERSITY IN ST. LOUIS

1 BRookings Drive

ST. LOUIS, MO 63130

UNITED STATES

roberts@math.wustl.edu 


\title{
PACIFIC JOURNAL OF MATHEMATICS
}

\author{
msp.org/pjm
}

Founded in 1951 by E. F. Beckenbach (1906-1982) and F. Wolf (1904-1989)

\section{EDITORS}

Don Blasius (Managing Editor)

Department of Mathematics

University of California

Los Angeles, CA 90095-1555

blasius@math.ucla.edu

\author{
Paul Balmer \\ Department of Mathematics \\ University of California \\ Los Angeles, CA 90095-1555 \\ balmer@math.ucla.edu \\ Robert Finn \\ Department of Mathematics \\ Stanford University \\ Stanford, CA 94305-2125 \\ finn@math.stanford.edu \\ Sorin Popa \\ Department of Mathematics \\ University of California \\ Los Angeles, CA 90095-1555 \\ popa@math.ucla.edu
}

\author{
Vyjayanthi Chari \\ Department of Mathematics \\ University of California \\ Riverside, CA 92521-0135 \\ chari@math.ucr.edu \\ Kefeng Liu \\ Department of Mathematics \\ University of California \\ Los Angeles, CA 90095-1555 \\ liu@math.ucla.edu \\ Jie Qing \\ Department of Mathematics \\ University of California \\ Santa Cruz, CA 95064 \\ qing@ cats.ucsc.edu
}

\section{PRODUCTION}

Silvio Levy, Scientific Editor, production@msp.org

\section{SUPPORTING INSTITUTIONS}

ACADEMIA SINICA, TAIPEI

CALIFORNIA INST. OF TECHNOLOGY

INST. DE MATEMÁTICA PURA E APLICADA

KEIO UNIVERSITY

MATH. SCIENCES RESEARCH INSTITUTE

NEW MEXICO STATE UNIV.

OREGON STATE UNIV.

\author{
STANFORD UNIVERSITY \\ UNIV. OF BRITISH COLUMBIA \\ UNIV. OF CALIFORNIA, BERKELEY \\ UNIV. OF CALIFORNIA, DAVIS \\ UNIV. OF CALIFORNIA, LOS ANGELES \\ UNIV. OF CALIFORNIA, RIVERSIDE \\ UNIV. OF CALIFORNIA, SAN DIEGO \\ UNIV. OF CALIF., SANTA BARBARA
}

\author{
Daryl Cooper \\ Department of Mathematics \\ University of California \\ Santa Barbara, CA 93106-3080 \\ cooper@math.ucsb.edu \\ Jiang-Hua Lu \\ Department of Mathematics \\ The University of Hong Kong \\ Pokfulam Rd., Hong Kong \\ jhlu@maths.hku.hk \\ Paul Yang \\ Department of Mathematics \\ Princeton University \\ Princeton NJ 08544-1000 \\ yang@math.princeton.edu
}

These supporting institutions contribute to the cost of publication of this Journal, but they are not owners or publishers and have no responsibility for its contents or policies.

See inside back cover or msp.org/pjm for submission instructions.

The subscription price for 2015 is US \$420/year for the electronic version, and \$570/year for print and electronic.

Subscriptions, requests for back issues and changes of subscribers address should be sent to Pacific Journal of Mathematics, P.O. Box 4163, Berkeley, CA 94704-0163, U.S.A. The Pacific Journal of Mathematics is indexed by Mathematical Reviews, Zentralblatt MATH, PASCAL CNRS Index, Referativnyi Zhurnal, Current Mathematical Publications and Web of Knowledge (Science Citation Index).

The Pacific Journal of Mathematics (ISSN 0030-8730) at the University of California, c/o Department of Mathematics, 798 Evans Hall \#3840, Berkeley, CA 94720-3840, is published twelve times a year. Periodical rate postage paid at Berkeley, CA 94704, and additional mailing offices. POSTMASTER: send address changes to Pacific Journal of Mathematics, P.O. Box 4163, Berkeley, CA 94704-0163.

PJM peer review and production are managed by EditFLOW ${ }^{\circledR}$ from Mathematical Sciences Publishers.

\section{PUBLISHED BY}

\section{mathematical sciences publishers \\ nonprofit scientific publishing}

http://msp.org/

(C) 2015 Mathematical Sciences Publishers 


\section{PACIFIC JOURNAL OF MATHEMATICS}

Volume $273 \quad$ No. $2 \quad$ February 2015

Taut foliations in surface bundles with multiple boundary components

TEJAS KALELKAR and RACHEL ROBERTS

Some results on archimedean Rankin-Selberg integrals

JINGSONG CHAI

Prescribing the boundary geodesic curvature on a compact scalar-flat Riemann

307

surface via a flow method

\section{HONG ZHANG}

-1-Phenomena for the pluri $\chi_{y}$-genus and elliptic genus

PING LI

On the geometry of Prüfer intersections of valuation rings

BRUCE OLBERDING

Non-Kähler expanding Ricci solitons, Einstein metrics, and exotic cone structures

Maria Buzano, Andrew S. Dancer, Michael Gallaugher and MCKENZIE WANG

A note on $L$-packets and abelian varieties over local fields

JEFFREY D. ACHTER and CLIFTON CUNNINGHAM

Rotating drops with helicoidal symmetry

Bennett Palmer and Oscar M. Perdomo

The bidual of a radical operator algebra can be semisimple

Charles John READ

Dimension jumps in Bott-Chern and Aeppli cohomology groups

JIEZHU LIN and XUANMING YE

Fixed-point results and the Hyers-Ulam stability of linear equations of higher orders

BING XU, JANUSZ BRZDĘK and WEINIAN ZHANG

Complete curvature homogeneous metrics on $\mathrm{SL}_{2}(\mathbb{R})$ 